\title{
Proposta de malha Stretched para ser utilizada com o método de imposição de dados discretos como condição de contorno de velocidade prescrita na geração numérica de ondas
}

Proposal of Stretched mesh to be used with the method of imposition of discrete data as boundary condition of prescribed velocity on numerical wave generation

\author{
E. V. Kisner ${ }^{1 *}$; B. N. Machado ${ }^{1}$; E. D. dos Santos ${ }^{1}$; L. A. O. Rocha ${ }^{2}$; M. N. \\ Gomes $^{3} ;$ L. A. Isoldi ${ }^{1}$ \\ ${ }^{1}$ Universidade Federal do Rio Grande (FURG), 96201-900, Rio Grande - RS, Brasil. \\ ${ }^{2}$ Universidade do Vale do Rio dos Sinos (Unisinos), 93022-750, São Leopoldo - RS, Brasil. \\ ${ }^{3}$ Institudo Federal do Paraná (IFPR), 83215-750, Paranaguá - PR, Brasil.
}

*elissandrokisner@furg.br

\begin{abstract}
O presente trabalho tem como objetivo avaliar duas possíveis soluções para um problema de convergência encontrado ao utilizar a malha Stretched, que basicamente é uma malha com refino localizado em regiões de interesse, juntamente com o método de imposição de dados discretos de velocidade como condição de contorno para a geração numérica de ondas. Realizou-se a análise computacional em um canal de ondas, em escala real, utilizando uma malha dividida em três zonas (estruturada com quadriláteros, não estruturada com triângulos e Stretched com quadriláteros) e uma malha dividida em duas zonas (não estruturada com triângulos e Stretched com quadriláteros). Para verificar estes modelos computacionais foram realizadas comparações da elevação da superfície livre, utilizando como base a onda gerada por metodologia já consolidada que impõe a condição de contorno de velocidade prescrita através de funções transientes das componentes de velocidade da onda. A modelagem computacional foi desenvolvida no software FLUENT, que é baseado no Método dos Volumes Finitos (MVF). O modelo multifásico Volume of Fluid (VOF) foi adotado para tratar adequadamente a interação entre ar e água. Os resultados obtidos mostraram que ambas as soluções propostas foram satisfatórias, permitindo a utilização de malha Stretched associada à imposição de dados discretos de velocidade prescrita para a geração numérica de ondas.

Palavras-chave: malha Stretched,energia das ondas, técnicas de geração numérica de ondas.
\end{abstract}

The present work aims to evaluate two possible solutions to a convergence problem found when using the Stretched mesh, which is basically a refined mesh located in regions of interest, along with the method of imposing discrete velocity data as boundary condition for the numerical generation of waves. Computational analysis was performed on a real-scale wave channel using a three-zone mesh (structured with quadrilaterals, unstructured with triangles and Stretched with quadrilaterals) and a mesh divided into two zones (unstructured with triangles and Stretched with quads). In order to verify these computational models, comparisons of the free surface elevation were made, using as basis the wave generated by already consolidated methodology that imposes the prescribed velocity boundary condition through the transient functions of the wave velocity components. The computational modelling was developed in FLUENT software, which is based on the Finite Volume Method (FVM). The multiphase Volume of Fluid (VOF) model was adopted to adequately treat the interaction between air and water. The results showed that both proposed solutions were satisfactory, allowing the use of Stretched mesh associated with the imposition of discrete velocity data for the numerical wave generation.

Keywords: Stretched mesh, wave energy, numerical wave generation tecniques.

\section{INTRODUÇÃO}

Tendo em vista o crescimento populacional e o aumento da preocupação com as questões ambientais, cresce o interesse por meios de produção de energia com baixo impacto e melhor rendimento. Uma importante ferramenta para o desenvolvimento de dispositivos para conversão da energia das ondas do mar em energia elétrica é a modelagem computacional. Além de fornecer resultados confiáveis, elimina a necessidade de construção de múltiplos protótipos para obtenção 
de dados de rendimento; aliando a produtividade, indispensável no cenário mundial de alta competitividade com a redução de refugos do processo e recursos financeiros despendidos.

Visando a realização da simulação numérica destes dispositivos, foram desenvolvidas técnicas de geração de ondas, como as citadas em Neves et al. (2010) [1], que vem passando por aprimoramentos ao longo dos anos, tais como:

- Imposição de condições de contorno, por meio da compilação de funções transientes para as componentes de velocidade da onda, que não estão implementadas originalmente no software, permitindo que a onda seja numericamente gerada. No software FLUENT essa metodologia é chamada de User-Defined Function (UDF), e é associada ao modelo Volume of Fluid (VOF), para tratar a interação água e ar, permitindo assim a geração da onda. Essa metodologia já foi utilizada em diversos trabalhos, como por exemplo em Gomes et al. (2017) [2].

- Em Machado et al. (2017) [3] foi apresentada uma variação do modelo anterior, tanto em escala de laboratório quanto em escala real, onde foi desenvolvida uma metodologia de imposição de dados tabelados como condição de contorno de velocidade prescrita para a geração da onda, que no software FLUENT é chamada de Table Data. Este modelo tem como vantagem a possibilidade de imposição de dados realísticos de estado de mar, que não possam ser adequadamente representados por uma função transiente.

- AMAZON: Baseia-se na resolução de equações de águas rasas (NLSW), que são simplificações das equações de Reynolds. Simula ondas aleatórias, além de utilizar uma condição de contorno de uma onda não refletiva que elimina $98 \%$ da energia de qualquer onda refletida de estruturas modeladas. Apenas em torno de 2010 foi iniciada a validação desde método para superfícies porosas, visto que sua forma original não explicitava valores para fluxos porosos. $\mathrm{Hu}$ (2000) [4] e Reis et al. $(2008,2009)$ [5, 6] apresentam uma descrição detalhada do modelo AMAZON.

- COBRAS/COBRAS-UC: A partir da média de volume das equações RANS (Reynolds Average Navier Stokes), Lin e Liu (1998) [7] desenvolveram um modelo matemático bidimensional para descrever o escoamento dentro e fora de estruturas costeiras, incluindo perfis porosos. Mais tarde foram incluídos pacotes de equações para o volume-médio e para o modelo de turbulência k-E. Utiliza o modelo VOF para descrever a interação entre água e ar. Já o COBRAS-UC é uma versão feita pela Universidade de Cantabria para superar algumas limitações do modelo original e convertê-lo para uso prático.

- SPHysics: Inicialmente utilizado em astrofísica, depois em simulações hidrodinâmicas e finalmente em aplicações costeiras. O SPH (Smoothed Particle Hydrodynamics) baseia-se em um método de partículas que dispensa o uso de malha, tendo uma abordagem lagrangeana. Devido a essa abordagem, condições de contorno móveis são facilmente implementadas. O modo de malha livre trata o modelo como um conjunto de partículas, representando uma porção de água. Monaghan (1992) [8] e Didier (2010) [9] apresentam de forma mais detalhada este método.

O presente trabalho utilizará os dois primeiros procedimentos numéricos de geração de ondas apresentados. Sabe-se que uma maneira de melhorar a qualidade dos resultados e diminuir o tempo de processamento na simulação numérica realizada no FLUENT é através da utilização de uma malha Stretched, como em Gomes et al. (2015) [10]. Essa técnica de geração de malha consiste em adotar malhas refinadas em regiões de interesse e malhas mais grosseiras nas demais regiões do domínio. Porém, em Machado et al. (2017) [3] foi empregada uma malha estruturada regular, uma vez que a malha Stretched causou a não convergência do modelo computacional, devido à obtenção de valores altos para os resíduos da equação de conservação da massa.

Sendo assim, o objetivo deste trabalho é realizar a união do método apresentado em Machado et al. (2017) [3] com a malha Stretched, permitindo que futuramente dados realísticos de estado de mar sejam simulados em um canal de ondas discretizado com malha Stretched. 


\section{MATERIAL E MÉTODOS}

A metodologia baseia-se na realização de dois estudos numéricos, um utilizando a malha de três regiões (estruturada com quadriláteros, não estruturada com triângulos e Stretched com quadriláteros) e outro com duas zonas (não estruturada com triângulos e Stretched com quadriláteros). Em ambos estudos será utilizado o método de imposição via Table Data no FLUENT, que permite a imposição de dados discretos de velocidade ao longo do tempo.

Os resultados obtidos foram então comparados com resultados de referência, gerados numericamente com metodologia já consagrada que utiliza uma malha puramente Stretched com quadriláteros. Esses resultados de referência são obtidos através do método de imposição de condição de contorno de velocidade prescrita via UDF no FLUENT, visto que essa malha aliada a UDF não gera problemas de convergência.

Para isso, as ondas utilizadas nesse trabalho possuem altura de $H=1,00 \mathrm{~m}$, período de $T=7,50$ $\mathrm{s}$ e comprimento de onda de $\lambda=65,40 \mathrm{~m}$, sendo geradas em um tanque com $h=10 \mathrm{~m}$ de profundidade de água assim como a onda gerada em Machado et al. (2017) [3].

O domínio computacional consiste em um canal de ondas bidimensional (Fig. 1) com altura de $H c=20 \mathrm{~m}$ e comprimento de $L c=327 \mathrm{~m}$. A Fig. 2 mostra também as condições de contorno adotadas para a modelagem computacional do canal de ondas: velocidade prescrita (Velocity Inlet, linha vermelha à esquerda); pressão atmosférica (Pressure Outlet, linha tracejada azul na esquerda e na região superior); e condição de impermeabilidade e não deslizamento (Wall, linha cinza na direita e na região inferior). Foram adotadas as recomendações mostradas em Machado et al. (2017) [3] quanto ao número das discretizações na zona de imposição de condição de contorno de velocidade prescrita (linha vermelha).

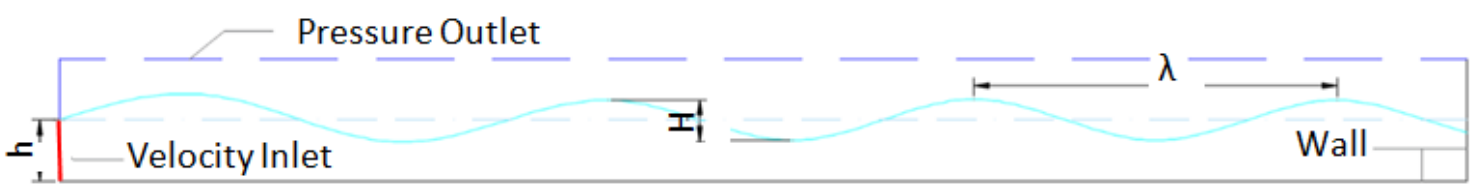

Figura 1: Domínio computacional utilizado.

Além disso, em uma primeira tentativa, o domínio computacional foi todo discretizado com malha Stretched com quadriláteros, como indicado em Gomes et al. (2017) [2]. Porém, aqui a imposição de velocidade prescrita foi realizada com Table Data e não com UDF. Assim, com a imposição de dados discretos enfrentou-se um erro de alta continuidade, acima de $1 \times 10^{-3}$. Tal resultado acarreta em um erro acumulado significativo, diminuindo consideravelmente a precisão dos estudos que seriam obtidos a partir deste modelo. Os valores dos resíduos para a equação de conservação da massa ou continuidade (linha branca) e para as equações de conservação de quantidade de movimento (linhas vermelha e verde), ao longo da simulação numérica, podem ser vistos na Figura 2. Todas as simulações realizadas neste trabalho, incluindo as malhas com duas e três zonas que serão apresentadas a seguir, possuem um passo de tempo de $0,05 \mathrm{~s}$ (de acordo com o utilizado em Machado et al. (2017) [3]) e tempo total de $100 \mathrm{~s}$. 


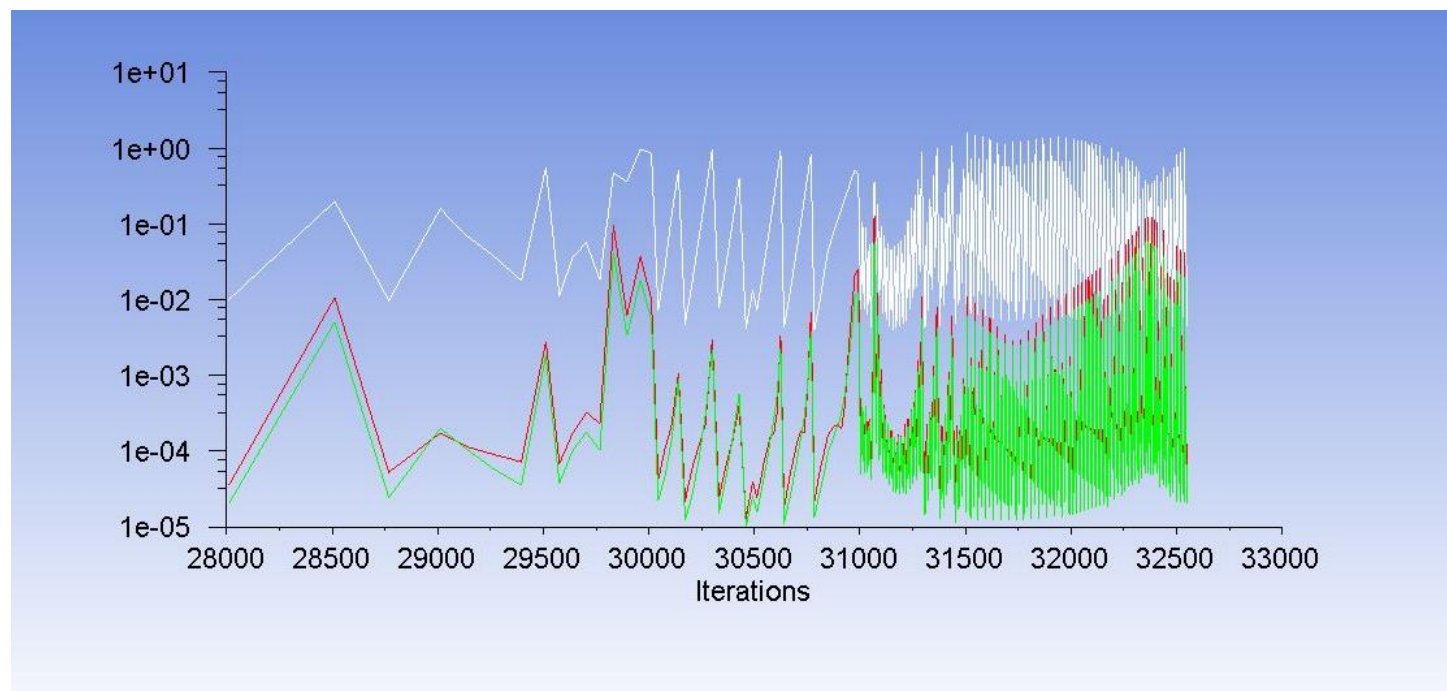

Figura 2: Resíduos obtidos com a utilização de Table Data e malha Stretched (gerado no FLUENT).

Em uma primeira tentativa de resolver o problema de convergência observado na Fig. 2, fez-se a proposta com a subdivisão do domínio computacional em três zonas com malhas diferentes, indicadas por linhas verticais na Figura 3. Na Figura 4, um detalhamento mostrando a zona $1 \mathrm{com}$ malha estruturada com quadriláteros, a zona 2 com malha não estruturada com triângulos e a zona 3 com malha Stretched com quadriláteros. Cabe destacar que esse tipo de divisão do domínio em regiões com malhas diferentes já foi utilizado em Grimmler et al. (2013) [11].

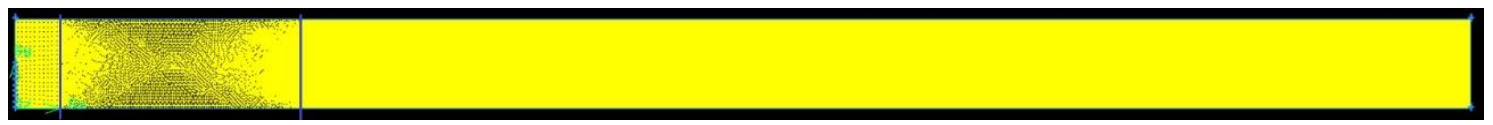

Figura 3: Domínio computacional discretizado em três zonas.

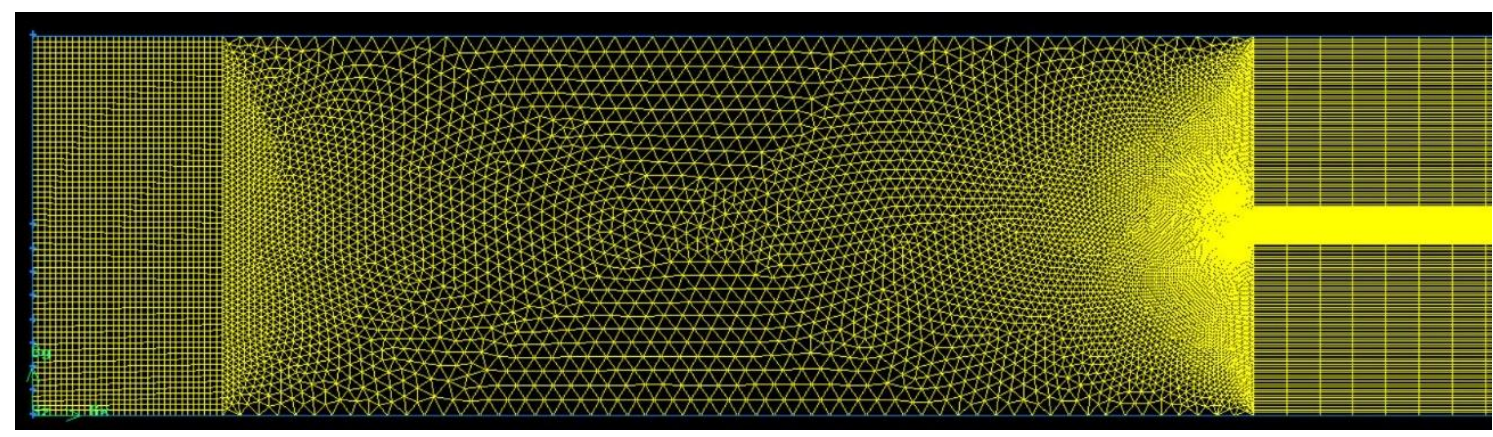

Figura 4: Visão detalhada da malha dividida em três zonas.

As malhas nas zonas 1 e 3 foram previamente geradas. Para a zona 1 foi utilizada uma discretização de $0,30 \mathrm{~m}$ nas direções horizontal e vertical. Na zona 3 utilizou-se uma malha Stretched com 150 divisões na direção horizontal, já na direção vertical tem-se 60 divisões acima e abaixo da região de superfície livre e 40 divisões na própria região de superfície livre, conforme Gomes et al. (2017) [2]. E para a discretização espacial da zona 2 utilizou-se como único parâmetro a dimensão máxima de $1 \mathrm{~m}$ para as células computacionais, deixando que a malha gerada se adaptasse às malhas das zonas 1 e 3 , previamente geradas.

Em um segundo momento foi feita a mesma simulação, porém utilizando a proposta com apenas 2 zonas na malha, suprimindo a zona com malha estruturada com quadriláteros do começo do canal de ondas (ver Figura 3). Então, nesse caso, a zona 1 é formada por malha não estruturada com 
triângulos e a zona 2 por malha Stretched com quadriláteros, como mostrado no detalhe na Figura 5.

Para tal foi gerada previamente a malha da zona 2, com as mesmas características citadas anteriormente para a malha Stretched. Após, a linha vertical esquerda do domínio computacional foi discretizada com segmentos de $0,30 \mathrm{~m}$. Então, a malha na zona 1 foi gerada, adaptando-se às discretizações previamente realizadas na zona 2 e na linha vertical.

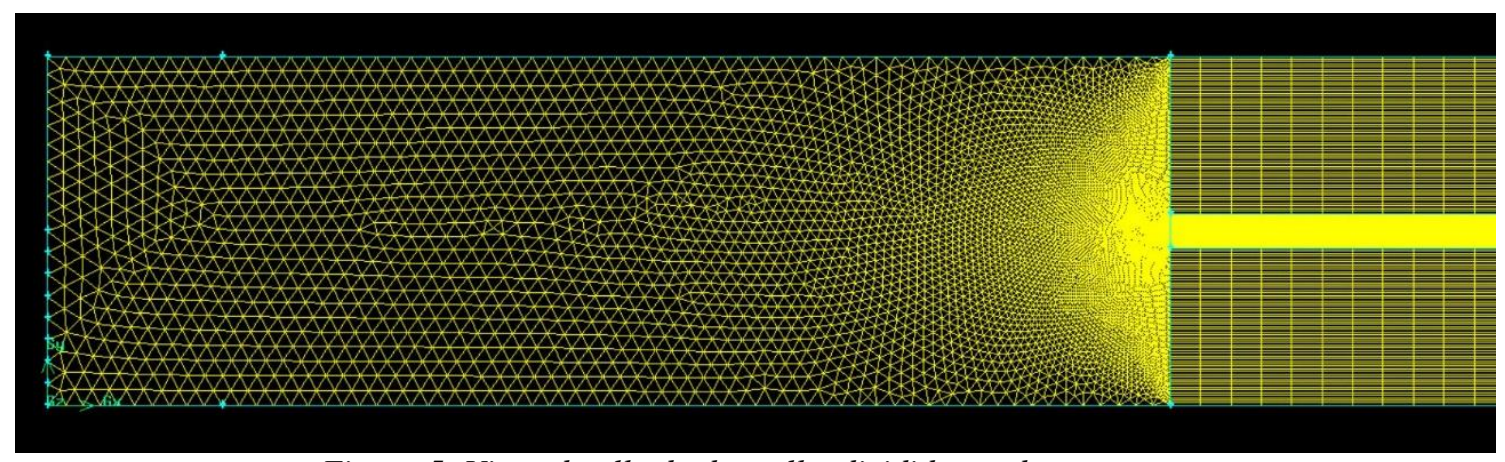

Figura 5: Visão detalhada da malha dividida em duas zonas.

Após a realização das simulações foi feita a comparação quanto à elevação da superfície livre medida a partir do fundo do tanque, assim como, se houve redução nos valores dos resíduos, principal objetivo deste trabalho.

A discretização do domínio computacional foi realizada no software GAMBIT, e as simulações no programa FLUENT, que é baseado no Método de Volumes Finitos (MVF). O método Volume of Fluid (VOF) foi empregado, o qual permite tratar adequadamente da interação entre água e ar [12].

\section{MODELAGEM MATEMÁTICA E SIMULAÇÃO NUMÉRICA}

Assim como mencionado, foi realizada uma simulação utilizando UDF para fins de comparação. A onda a ser gerada, com características anteriormente mencionadas, é classificada como de Stokes de $2^{\mathrm{a}}$ Ordem, sendo assim as condições impostas a superfície de entrada do domínio são dadas por:

$$
\begin{gathered}
u=A g k \frac{\cosh (k z+k h)}{\omega \cosh (k h)} \cos (k x-\omega t)+A^{2} \omega K \frac{\cosh 2 k(h+z)}{\operatorname{sen}^{4}(k h)} \cos 2(k x-\omega t) \\
w=A g k \frac{\operatorname{senh}(k z+k h)}{\omega \operatorname{senh}(k h)} \operatorname{sen}(k x-\omega t)+A^{2} \omega K \frac{\operatorname{senh} 2 k(h+z)}{\cos ^{4}(k h)} \operatorname{sen} 2(k x-\omega t)
\end{gathered}
$$

onde: $A$ é a amplitude da onda $(\mathrm{m}) ; g$ a aceleração da gravidade $\left(\mathrm{m} / \mathrm{s}^{2}\right) ; L$ é o comprimento da onda, $k$ é o número de onda dado por $k=2 \pi / L\left(\mathrm{~m}^{-1}\right) ; T$ é o período da onda (s); $\omega$ é a frequência dada por $\omega=2 \pi / \mathrm{T}(\mathrm{Hz}) ; h$ é a profundidade $(\mathrm{m}) ; x$ é a posição espacial $(\mathrm{m}) ; t$ é o tempo (s) e $z$ é a variação da posição entre a superfície livre da água e o fundo do mar (m).

Já o movimento da superfície livre pode ser descrito por:

$$
\eta=\frac{H}{2} \cos (k x-\sigma t)+\frac{H^{2} k \operatorname{consh} k h}{16 \operatorname{senh}^{3} k h}(2+\cosh 2 k h) \cos 2(k x-\sigma t)
$$

Existe uma grande importância associada à Eq. (3) visto que, a partir dela, é possível realizar a verificação de modelos computacionais. As Eqs. (1), (2) e (3) são apresentadas em McCormick (1976) [13] e Dean e Dalrymple (1991) [14]. Maiores detalhes sobre a teoria de ondas oceânicas podem ser encontrados nessas referências.

O método Volume of Fluid (VOF), desenvolvido por Hirt e Nichols (1981) [15], é utilizado neste trabalho. É um modelo multifásico que se baseia na premissa de que o volume ocupado por uma fase não pode ser ocupado por outra. Sendo assim, utiliza-se o conceito de fração de volume $(\alpha)$. 
Além disso, ao utilizar o VOF apenas um conjunto de equações é aplicado a todos os fluidos componentes do escoamento. Sendo esse conjunto composto pela equação da continuidade (Eq. (4)) e pela equação da quantidade de movimento (Eq. (5)), além da própria equação da fração volumétrica (Eq. (6)).

$$
\begin{gathered}
\frac{\partial(\rho)}{\partial t}+\nabla \cdot(\rho \vec{v})=0 \\
\frac{\partial}{\partial t}(\rho \vec{v})+\nabla \cdot(\rho \vec{v} \vec{v})=-\nabla p+\nabla \cdot(\mu \dot{\tau})+\rho \vec{g} \\
\frac{\partial(\alpha)}{\partial t}+\nabla \cdot(\alpha \vec{v})=0
\end{gathered}
$$

onde: $\rho$ é a massa específica do fluido $\left(\mathrm{kg} / \mathrm{m}^{3}\right), t$ é o tempo (s), $\vec{v}$ é o vetor velocidade do escoamento $(\mathrm{m} / \mathrm{s}), p$ é a pressão estática $\left(\mathrm{N} / \mathrm{m}^{2}\right), \mu$ é a viscosidade $(\mathrm{kg} /(\mathrm{ms})), t$ é o tensor de tensões $\left(\mathrm{N} / \mathrm{m}^{2}\right)$ e $\vec{g}$ é a aceleração da gravidade $\left(\mathrm{m} / \mathrm{s}^{2}\right)$.

É necessário então, calcular os valores médios para massa específica (Eq. (7)) e viscosidade (Eq. (8)) Srinivasan et al. (2010) [16]:

$$
\begin{aligned}
& \rho=\alpha_{\text {água }} \rho_{\text {água }}+\alpha_{\text {ar }} \rho_{a r} \\
& \mu=\alpha_{\text {água }} \mu_{\text {água }}+\alpha_{\text {ar }} \mu_{a r}
\end{aligned}
$$

\section{RESULTADOS E DISCUSSÃO}

As malhas das Figuras 4 e 5 foram então utilizadas em simulações numéricas para a geração de ondas regulares. Ambas as discretizações propostas reduziram consideravelmente o valor dos resíduos para a conservação da massa (continuidade) e para as componentes da quantidade de movimento (velocidades nas direções $x$ e $z$ ). A convergência foi obtida para valores de resíduos inferiores a $1 \times 10^{-4}$, o que representa um resultado satisfatório para a finalidade deste trabalho.

Além disso, ambas as simulações tiveram aproximadamente o mesmo comportamento, levando em consideração que estudos mais aprofundados sobre a malha e tempo de simulação serão realizados posteriormente.

Na Figura 6, utilizando a malha com três regiões, é possível notar que variação do valor resíduo da continuidade (linha branca) apresenta um comportamento com melhor convergência se comparado à Figura 2.

Na Figura 7 percebe-se uma ligeira diferença nos resíduos de velocidade (linhas verde e vermelha) utilizando a malha de duas zonas, em relação à Figura 6. Porém, como será mostrado na sequência isso não causou diferença significativa na elevação da superfície livre, a partir do fundo do tanque, entre as duas soluções apresentadas. 


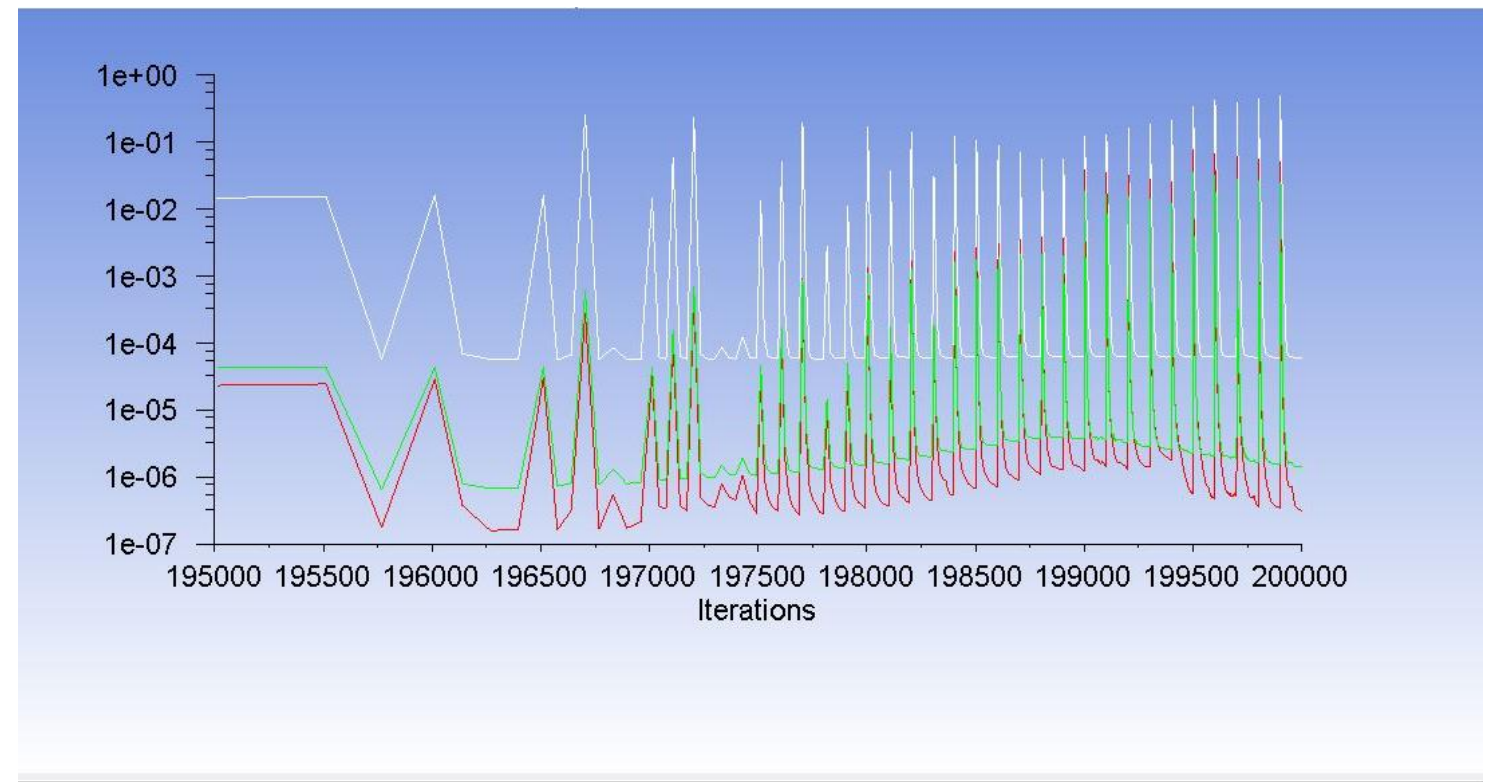

Figura 6: Resíduos utilizando a malha com três regiões (gerado no FLUENT).

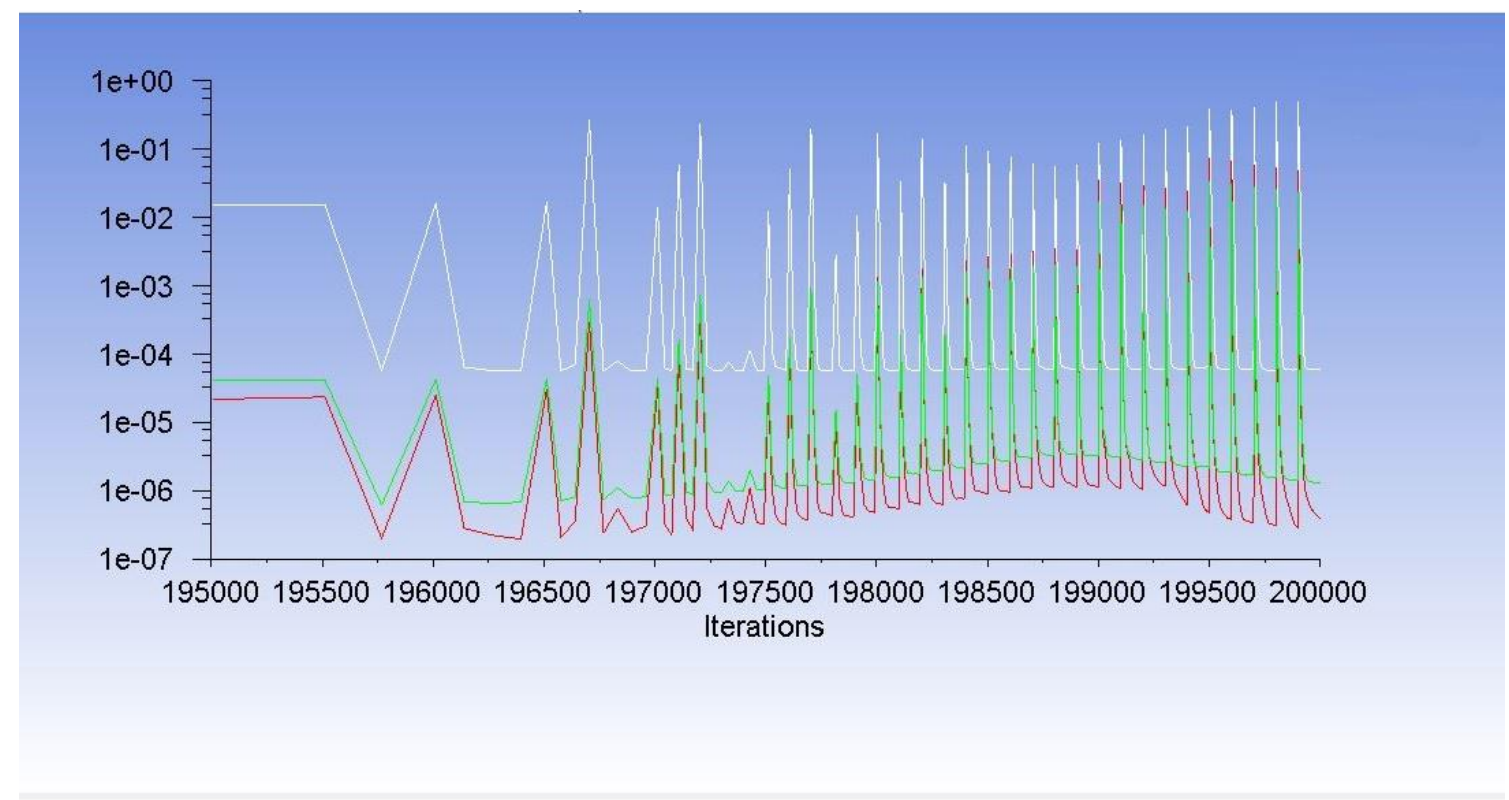

Figura 7: Resíduos utilizando a malha com duas regiões (gerado no FLUENT).

Quanto aos valores de elevação da superfície livre, outro parâmetro levado em consideração para fins de comparação, ambas as soluções reproduziram com diferença muito pequena a onda produzida por meio de UDF com malha totalmente Stretched com quadriláteros, como pode-se observar na Figura 8, onde o eixo vertical representa a elevação da superfície livre, medida em relação ao fundo do tanque, e o eixo horizontal o tempo. 


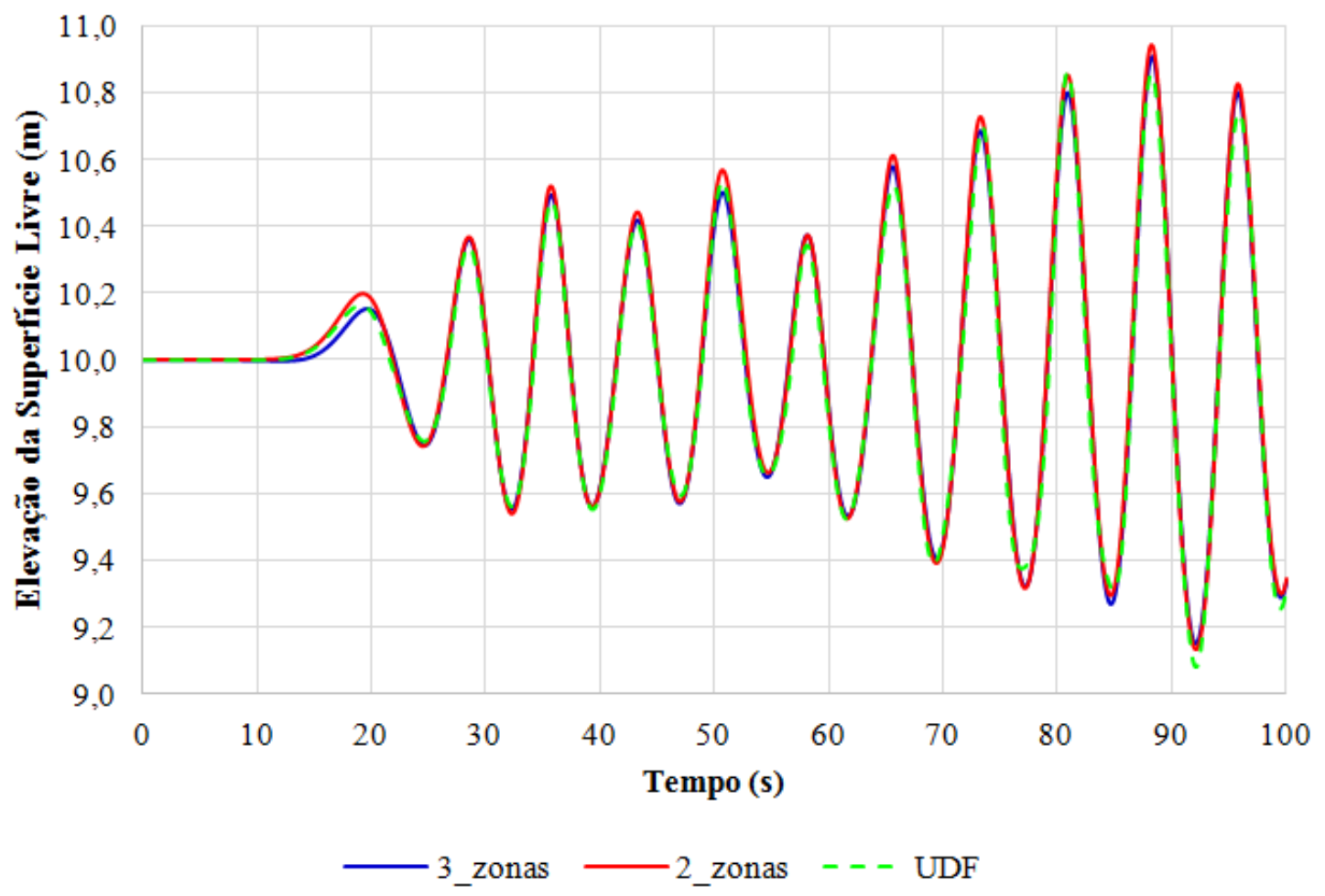

Figura 8: Comparação da elevação da superfície livre.

Como pode ser visto na Tabela 1 os erros relativos simples para os resultados numéricos obtidos com as malhas com duas e três zonas simuladas com a metodologia Table Data em relação ao resultado utilizando a UDF em malha totalmente Stretched com quadriláteros é muito pequeno, mesmo sem a realização de estudos de independência de malha e passo de tempo.

Tabela 1: Erro relativo máximo em relação a UDF.

\begin{tabular}{cc}
\hline Número de zonas da malha & $\mid \boldsymbol{\%}$ MaxVar $\mid=(\boldsymbol{a}-\boldsymbol{b}) / \boldsymbol{a} * \mathbf{1 0 0}$ \\
\hline 3_zonas & 0,96 \\
2_zonas & 0,88 \\
\hline
\end{tabular}

Comparando as soluções com três e duas zonas entre si foi obtido um erro relativo máximo de $0,61 \%$, lembrando que não foram realizados testes de convergência de malha para nenhuma das duas soluções.

A partir das comparações mostradas é possível observar que, para o que foi proposto neste trabalho, os resultados foram satisfatórios.

\section{CONCLUSÃO}

A contribuição principal do trabalho apresentado foi a realização de duas propostas de malha para resolver o problema de convergência encontrado ao utilizar a malha Stretched juntamente com o método Table Data.

Ambas as discretizações propostas permitiram melhorar significativamente a convergência dos resultados numéricos para a geração de ondas regulares.

Os resultados deste trabalho são promissores em relação ao uso das malhas propostas para a geração de ondas que representem um estado de mar realístico, empregando dados discretos de velocidade prescrita como condição de contorno em um canal de ondas com malha Stretched. 
Em trabalhos futuros estudos de convergência de malha e de convergência de passo de tempo devem ser realizados, visando aprimorar ainda mais os resultados obtidos. Então, com isso, usar essas recomendações de discretização espacial de canal de ondas para o estudo do comportamento fluidodinâmico de dispositivos conversores de energia das ondas do mar submetidos a estados de mar realísticos.

\section{AGRADECIMENTOS}

Os autores agradecem à Fundação de Amparo à Pesquisa do Rio Grande do Sul (FAPERGS) e ao Conselho Nacional de Desenvolvimento Científico e Tecnológico (CNPq) pelo apoio financeiro à realização dessa pesquisa. $\mathrm{O}$ autor $\mathrm{E}$. V. Kisner agradece à FAPERGS pela bolsa de Iniciação Científica. O autor L. A. Isoldi agradece a FAPERGS pelo recurso aprovado no Edital 02/2017 PqG (Processo: 17/2551-0001111-2). Os autores E. D. dos Santos, L. A. Isoldi e L. A. O. Rocha agradecem ao CNPq por suas bolsas de Produtividade em Pesquisa (Processos: 306024/2017-9, 306012/2017-0 e 307847/2015-2, respectivamente).

\section{REFERÊNCIAS BIBLIOGRÁFICAS}

1. Neves MG, Reis MT, Didier E. Comparisons of wave overtopping at coastal structures calculated with AMAZON, COBRAS-UC and SPHYSICS. V European Conference on Computational Fluid Dynamics. 2010 Jun.

2. Gomes MN, Isoldi, LA, Dos Santos ED, Rocha LAO. Simulação numérica de um dispositivo coluna de água oscilante em grandes intervalos de tempo. XX Encontro Nacional de Modelagem Computacional (ENMC). Anais do XX ENMC, 2017. Nova Friburgo, RJ. UERJ.

3. Machado BN, Kisner EV, Paiva MS, Gomes MN, Rocha LAO, Marques WC, dos Santos ED, Isoldi LA. Numerical generation of regular waves using discrete analytical data as boundary condition of prescribed velocity. XXXVIII Iberian-Latin American Congress on Computational Methods in Engineering. 2017 Nov, doi:10.20906/CPS/CILAMCE2017-0816

4. Hu K. High-resolution finite volume methods for hydraulic flow modelling [thesis]. Center for Mathematical Modelling and Flow Analysis, Manchester Metropolitan University; 2000.

5. Reis MT, Hu K, Neves MG, Hedges TS. Numerical modelling of breakwater overtopping using a NLSW equation model with a porous layer. Coastal Eng 2008. 2009:3097-3109, doi: 10.1142/9789814277426_0256

6. Reis M, Neves M, Hu K. Wave Overtopping of a Porous Structure: Numerical and Physical Modeling. J Coastal Res 2008. 2009:539-543.

7. Lin P, Liu P. A numerical study of breaking waves in the surf zone. J Fluid Mech. 1998;359:239-264, doi: $10.1017 /$ S002211209700846X

8. Monaghan JJ. Smoothed particle hydrodynamics. Ann Rev Astron Astrophys. 1992;30(1):543-574, doi: 10.1146/annurev.aa.30.090192.002551

9. Didier E, Neves M. Modelação da Interacção entre uma Onda e uma Estrutura de Proteç̧ão Costeira usando um Modelo Numérico SPH - Smoothed Particles Hydrodynamics. Rev Gestão Costeira Integr - J Integr Coastal Zone Manag. 2010;10(4):435-455.

10. Gomes MDN, Lara MFE, Iahnke SLP, Machado BN, Goulart, et al. Numerical approach of the main physical operational principle of several wave energy converters: oscillating water column, overtopping and submerged plate. Defect Diffus Forum. 2015;362:115-171, doi: 10.4028/www.scientific.net/DDF.362.115

11. Grimmler JDAM. Conversor de energia das ondas em energia elétrica com dispositivo de coluna de água oscilante: simulação numérica e estudo geométrico (Master's thesis). 2013.

12. Fluent Inc. FLUENT 12.0 Theory Guide. 2009.

13. McCormick ME. Ocean Engineering Mechanics. New York: Cambridge University Press. 1976.

14. Dean RG, Dalrymple RA. Water wave mechanics for engineers and scientists (vol. 2). World Scientific Publishing Company. 1991.

15. Hirt CW, Nichols BD. Volume of fluid (VOF) method for the dynamics of free boundaries. J Comput Phys. 1981;39(1):201-225, doi: 10.1016/0021-9991(81)90145-5

16. Srinivasan V, Salazar AJ, Saito K. Modeling the disintegration of modulated liquid jets using volume-offluid (VOF) methodology. Appl Mathem Model. 2011;35(8):3710-3730, doi: 10.1016/j.apm.2011.01.040 